\title{
Surface-Enhanced Raman Scattering in Purely Dielectric Structures via Bloch Surface Waves
}

\author{
S. Pirotta, ${ }^{\dagger}{ }^{\dagger}$ X. G. Xu, ${ }^{\S}$ A. Delfan, ${ }^{\|}$S. Mysore, ${ }^{\S}$ S. Maiti, ${ }^{\#}$ G. Dacarro, ${ }^{\dagger}$ M. Patrini, ${ }^{\dagger}$ M. Galli, ${ }^{\dagger}$ G. Guizzetti, ${ }^{\dagger}$ \\ D. Bajoni, ${ }^{\ddagger}$ J. E. Sipe, ${ }^{\|}$G. C. Walker, ${ }^{\perp}$ and M. Liscidini ${ }^{\dagger}$ \\ ${ }^{\dagger}$ Department of Physics and ${ }^{\ddagger}$ Dipartimento di Ingegneria Industriale e dell’nformazione, University of Pavia, Pavia, I-27100 Italy \\ ${ }^{\S}$ Department of Chemistry, "Department of Physics and Institute for Optical Science, and ${ }^{\perp}$ Department of Chemistry, University of \\ Toronto, Toronto, Canada \\ \#Department of Chemical Science, Tata Institute of Fundamental Research, Homi Bhabha Road, Colaba, Mumbai 400005, India
}

ABSTRACT: We study surface-enhanced Raman scattering (SERS) in fully dielectric periodic structures supporting Bloch surface waves. We demonstrate a lower bound for the SERS enhancement of 50 when the optical pump is resonantly coupled to the Bloch surface wave supported by the structure. A corresponding photoluminescence experiment shows an emission enhancement of more than two orders of magnitude, suggesting that the actual SERS enhancement is $\sim 200$. Analogous conclusions are obtained by means of a semiclassical theoretical model. These results suggest an alternative

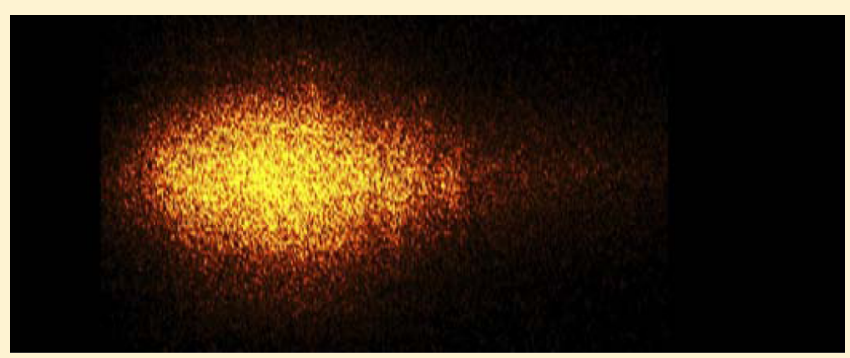
to plasmonic materials to enhance Raman scattering at a surface and are of interest to those working in the field of Raman as well as fluorescence spectroscopy.

\section{INTRODUCTION}

In the last 30 years, Raman spectroscopy has become one of the most utilized tools in optical sensing and biosensing. ${ }^{1,2}$ Indeed, the narrow line transitions of the vibrational bands of molecules give this technique high selectivity and the possibility of obtaining structural information of complex analytes, yet the Raman response of molecules is usually very weak, ${ }^{3,4}$ and thus strategies to increase the sensitivity of this powerful spectroscopic technique are needed. An approach often employed is the use of surface-enhanced Raman scattering (SERS), where the excitation of surface plasmons in roughened $\mathrm{d}^{5-7}$ or periodically structured metallic surfaces ${ }^{8,9}$ and metal nanoparticles ${ }^{10}$ can lead to a large field enhancement. Amplification of a Raman signal up to 14 orders of magnitude has been demonstrated in aggregated gold and silver nanoparticles, ${ }^{10}$ and more generally, enhancements from four to six orders of magnitude are commonly observed from metallic substrates.

Another approach to SERS consists of exploiting the resonant coupling of incident and scattered radiation with guided modes in dielectric structures. For instance, previous work has demonstrated up to three orders of magnitude enhancement in slab waveguides. ${ }^{11-15}$ Although purely dielectric structures do not seem to be able to compete with metallic structures in terms of the enhancement that can be achieved, the interest in the use of dielectric structures is motivated by the wide range of experimental configurations that can be implemented. For instance, photonic crystal fibers are particularly promising for in situ Raman spectroscopy of colloidal solutions. ${ }^{16}$ More generally, dielectric systems offer the possibility of working with transparent materials in the frequency range of interest, which is particularly appealing for the realization of integrated on-chip Raman sensors or microscope slides that could be mass produced for dark-field Raman imaging at interfaces.

Recently, it has been suggested that truncated periodic dielectric multilayers supporting Bloch surface waves (BSWs) might lead to SERS with up to five orders of magnitude of enhancement. ${ }^{17}$ In these systems, light is confined near the surface by total internal reflection from the upper cladding medium and by the photonic band gap (PBG) from the multilayer side. ${ }^{18}$ The characteristic that makes this approach particularly appealing is the possibility of working over a wide spectral range ${ }^{19,20}$ with several materials, from oxides ${ }^{17}$ to semiconductors, ${ }^{21}$ even when the refractive index contrast is very small. Moreover, starting from a truncated multilayer, it is possible to confine and guide light along specific directions, opening the path to the full integration of sensors based on this platform. $^{21-23}$

In this work we consider SERS in $\mathrm{Ta}_{2} \mathrm{O}_{5} / \mathrm{SiO}_{2}$ truncated periodic multilayers, and we analyze the Raman results with the aid of angle-resolved attenuated total reflectance (ATR), photoluminescence (PL) measurements, and a semiclassical theoretical model. We demonstrate that Raman scattering can be enhanced up to 2 orders of magnitude when the pump beam is coupled to the BSW in the Kretschmann configuration. Our

Received: January 8, 2013

Revised: March 4, 2013

Published: March 21, 2013 
theoretical analysis ${ }^{17}$ indicates the potential of even larger enhancements with these structures. SERS is observed in two different samples that have been designed to work with different pump wavelengths, demonstrating the applicability of this approach from the infrared to the visible spectral region.

The manuscript is organized as follows: In Section II, we discuss the multilayer structures and their linear characterization by means of angle-resolved ATR measurements. In Section III, we present Raman spectra for visible and infrared pumps, and we discuss the origin and the value of the enhancement by comparing these results with PL measurements and our theoretical model. Finally, in Section IV, we present our conclusions.

\section{MULTILAYER STRUCTURE AND CHARACTERIZATION}

A sketch of the typical structure of the samples investigated in this work is shown in Figure 1a. Each sample consists of a (a)

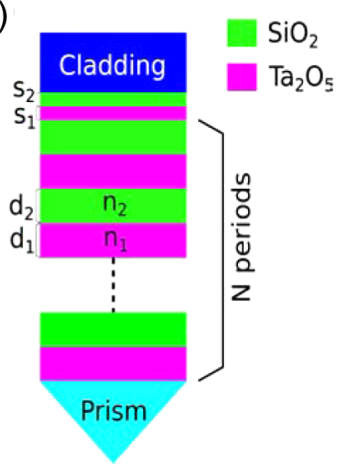

(b)
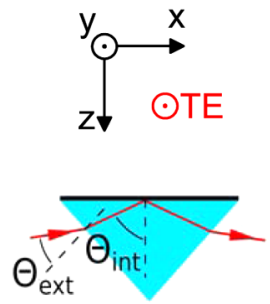

Figure 1. (a) Sketch of the typical multilayer supporting a Bloch surface wave. (b) Experimental configuration used in the angleresolved attenuated total reflectance spectra. The external and internal angles of incidence are indicated.

$\mathrm{Ta}_{2} \mathrm{O}_{5} / \mathrm{SiO}_{2}$ multilayer grown by magnetron sputtering on the hypotenuse of a 45-90-45 N-BK7 prism. The nominal parameters of IR and VIS structures are reported in Table 1.

\section{Table 1. Nominal Parameters of VIS and IR Samples}

\begin{tabular}{lccc} 
& VIS sample $\left(\lambda_{\text {pump }}=780 \mathrm{~nm}\right)$ & & IR sample $\left(\lambda_{\text {pump }}=1064 \mathrm{~nm}\right)$ \\
\cline { 2 - 2 } manufacturer & spectrum thin films & & iridian spectral technologies ${ }^{27}$ \\
$\mathrm{~s}_{1}\left(\mathrm{Ta}_{2} \mathrm{O}_{5}\right)$ & $50 \mathrm{~nm}$ & & $80 \mathrm{~nm}$ \\
$\mathrm{~s}_{2}\left(\mathrm{SiO}_{2}\right)$ & $10 \mathrm{~nm}$ & $10 \mathrm{~nm}$ \\
$\mathrm{~d}_{1}\left(\mathrm{Ta}_{2} \mathrm{O}_{5}\right)$ & $150 \mathrm{~nm}$ & $200 \mathrm{~nm}$ \\
$\mathrm{~d}_{2}\left(\mathrm{SiO}_{2}\right)$ & $150 \mathrm{~nm}$ & $250 \mathrm{~nm}$ \\
periods & 5 & 5 \\
\hline
\end{tabular}

Each multilayer stack is composed of five periods of a bilayer unit cell (thicknesses $d_{1}$ and $d_{2}$ of the $\mathrm{Ta}_{2} \mathrm{O}_{5}$ and $\mathrm{SiO}_{2}$ layers, respectively), with two additional layers on the top of the structure (thicknesses $s_{1}$ and $s_{2}$ of the $\mathrm{Ta}_{2} \mathrm{O}_{5}$ and $\mathrm{SiO}_{2}$ layers respectively, with the $\mathrm{SiO} 2$ layer closest to the cladding) to obtain the desired BSW dispersion following the procedure previously reported. ${ }^{19}$ We designed two different samples, one to support a BSW in the visible (VIS) and one to support a BSW in the infrared (IR) spectral regions, with the upper cladding being water and air, respectively. In both cases, we consider transverse-electric (TE) polarized BSWs, that is, electric field perpendicular to the plane of incidence (see Figure
1), but we stress that multilayers can be designed to support BSWs in either TE or transverse-magnetic (TM) polarization. ${ }^{19}$ The two samples were characterized by means of angle-resolved ATR measurements in the Kretschman configuration ${ }^{24}$ to verify the presence of BSWs and to map their dispersion relations. In this configuration (see Figure 1b), the excitation of a BSW is indicated by a dip in the reflectance due to the unavoidable scattering losses at the surface of the sample. ${ }^{25}$ Similar but larger dips can be observed in structures involving surface plasmons, where the losses are much larger and primarily due to absorption.

In Figure 2a, we show the TE ATR spectra obtained for the VIS sample when the upper cladding is water $(n \approx 1.33)$ at
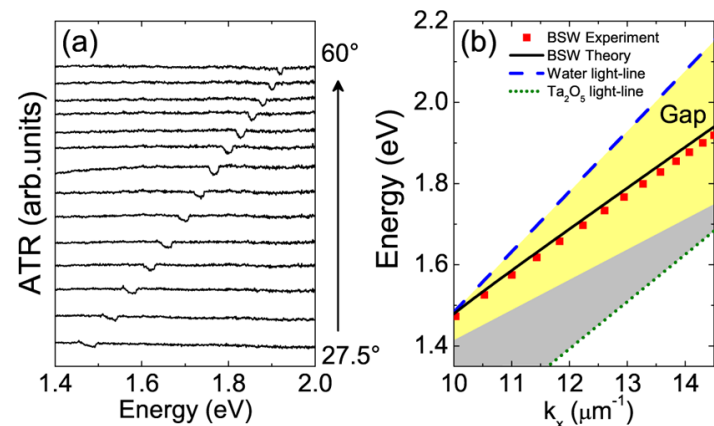

Figure 2. (a) TE angle-resolved attenuated total reflectance spectra of the VIS sample, curves are vertically shifted for clarity. (b) Calculated dispersion of the BSW (black solid) and BSW energies data obtained from the ATR measurements (red square) for the VIS sample. Water (blue dashed) and $\mathrm{Ta}_{2} \mathrm{O}_{5}$ (green dotted) light lines are also shown. The photonic band gap region between the two light lines is highlighted in yellow.

different external angles of incidence $\theta_{\text {ext }}$ from $27.5^{\circ}$ to $60^{\circ}$ with steps of $2.5^{\circ}$. These curves are obtained using a FTIR spectrometer (Bruker IFS 66/S) coupled to an external homemade microreflectometer equipped with a $\mathrm{Si}$ photodiode detector. The BSW dispersion curve calculated using the nominal parameters of Table 1 is shown in Figure $2 \mathrm{~b}$. As expected, the curve is within the PBG determined by the multilayer and below the water light line, which describes the photon dispersion relation in water. We find good agreement between the theoretical curve and the experimental points extracted from the dips in the ATR spectra of Figure 2a. A similar optical characterization has been performed on the IR sample.

Finally, in Figure 3, we show an optical image and the calculated intensity profile ${ }^{17}$ of the spot arising when the BSW is excited at the surface of the VIS sample with a TE-polarized pump at wavelength $\lambda=780 \mathrm{~nm}$ and external angle of incidence $\theta_{\text {ext }}=31^{\circ}$. Along the $y$ direction, the spot has a full width at half-maximum $\mathrm{FWHM}_{y}=479 \mu \mathrm{m}$, which is the same as the spot impinging the prism facet. Along the $x$ direction, the spot in the focus of the lens has $\mathrm{FWHM}_{x}$ of $265 \mu \mathrm{m}$, but on the multilayer surface it is elongated due to the finite angle of incidence on prism and with a comet-like shape corresponding to the excitation of the BSW.

\section{RAMAN SCATTERING RESULTS AND DISCUSSION}

In this work, we investigate Raman scattering from a substance nearby the sample surface in the experimental configuration depicted in Figure 4, where the pump beam is impinging from 

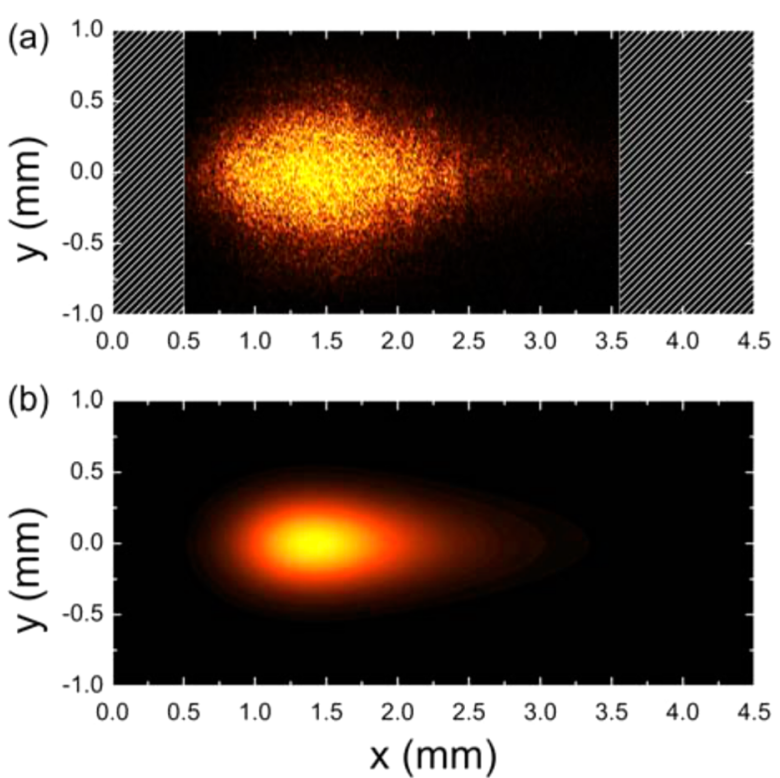

Figure 3. (a) Optical image on the surface of the VIS sample when the BSW is excited at $\lambda=780 \mathrm{~nm}$ and $\theta_{\text {ext }}=31^{\circ}$. (b) Calculated intensity profile using the sample nominal parameters. We consider a spot with $\mathrm{FWHM}_{y}=479 \mu \mathrm{m}$ and $\mathrm{FWHM}_{x}=265 \mu \mathrm{m}$.

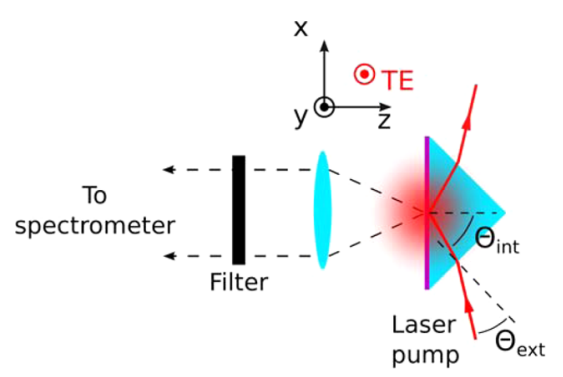

Figure 4. Sketch of the experimental configuration used in Raman scattering and PL measurements.

the prism facet and Raman scattering is detected from the top of the sample through a microscope objective. In this configuration, it is possible to observe SERS given by the excitation of BSWs by the pump beam. Here we work with a TE-polarized pump at wavelengths 780 and $1064 \mathrm{~nm}$ for the VIS and IR samples, respectively. We collect the total Raman scattering from the cladding side independently of the polarization.

a. VIS Sample. We consider the use of the VIS sample to detect Raman scattering arising from a solution in a slide-cell realized on the sample surface. When the cell is filled with a solution of acetone in distilled water $(4: 1 \mathrm{v} / \mathrm{v})$, having refractive index $n \approx 1.33$, the VIS sample supports a BSW that can be excited at $\lambda_{\text {pump }} \approx 780 \mathrm{~nm}$ at the external angle $\theta_{\text {ext }}=31^{\circ}$. We use a continuous-wave (CW) laser diode (Thorlabs L785P090) as our source; the beam is focused on the sample by means of a $25 \mathrm{~cm}$ focal-length lens, with $\sim 50 \mathrm{~mW}$ power on the sample. The Stokes Raman signal is collected from the top of the sample through a microscope objective (Mitutoyo $5 \times$, NA = 0.14), and a Semrock $785 \mathrm{~nm}$ RazorEdge ultrasteep long-pass edge filter is used to remove the Rayleigh scattering contribution coming from the pump. Finally, the signal is sent to a monochromator (Acton SP-2300, Princeton Instruments) coupled to a liquid nitrogen-cooled Silicon CCD (Spec10 System, Princeton Instruments). To evaluate the enhance- ment value of the Raman signal, we consider a bare (that is, without the multilayer structure) N-BK7 prism (BP) with an identical slide-cell filled with the same solution as our reference. Raman spectra are also collected when the cells of VIS and BP are filled with pure water to measure the background Raman scattering contribution coming from the prism and the multilayer. (See the inset of Figure 5a.)
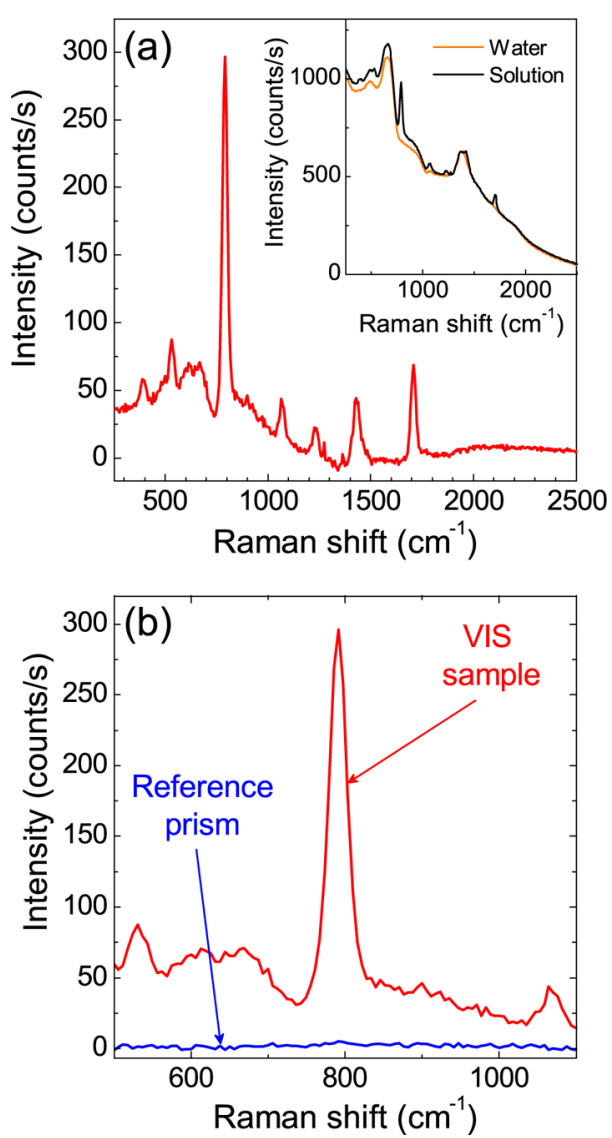

Figure 5. (a) (Inset) Raman spectra collected from the top of the VIS sample for water (background) and a solution composed of 1:4 v/v distilled $\mathrm{H}_{2} \mathrm{O}$ and acetone when the pump $\left(\lambda_{\text {pump }}=780 \mathrm{~nm}\right)$ is resonantly coupled to the $\mathrm{BSW}$ throughout the $\mathrm{N}-\mathrm{BK} 7$ prism. (Main panel) Raman spectrum after background subtraction. (b) Detail of the peak around $800 \mathrm{~cm}^{-1}$ and corresponding spectrum obtained for the bare prism after background subtraction.

In the main panel of Figure 5a, we show the Raman spectrum of the acetone solution for the VIS sample after background subtraction. In Figure 5b, we report a zoom of the spectrum around the acetone Raman peak at $\sim 800 \mathrm{~cm}^{-1}$ along with the signal detected from the BP structure. The absence of the acetone peak in the case of the bare prism does not allows us to estimate the corresponding enhancement due to the excitation of the BSW, yet it is still possible to give a lower bound of the Raman enhancement by taking the ratio between the intensity of the strongest acetone Raman peak and the noise amplitude of the BP measurement: the ratio is $\sim 50$.

We use the theoretical semiclassical model previously reported $^{17}$ to analyze the performance of the VIS structure, considering the bulk Raman contribution given by the entire solution subjected to the evanescent field associated with the excitation of the BSW. The expected enhancement curve as a function of the pump external angle of incidence is presented in 


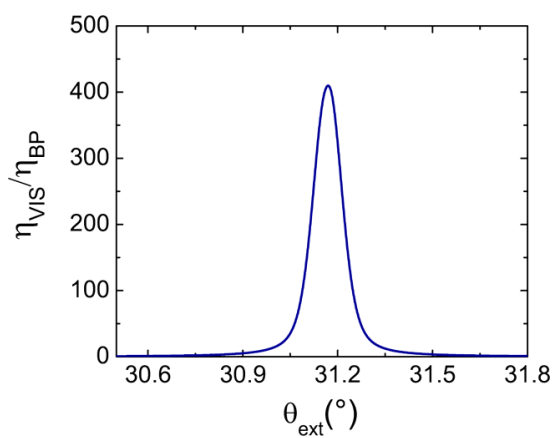

Figure 6. Calculated Raman enhancement of VIS with respect to BP as a function of the pump angle of incidence $\theta_{\text {ext }}$. We assume $\lambda_{\text {pump }}=780$ $\mathrm{nm}$ and Stokes shift of $800 \mathrm{~cm}^{-1}$.

Figure 6, where the maximum enhancement at resonance is $\sim 400$ and occurs when the BSW is excited. The calculation predicts an expected enhancement eight times larger than the lower bound of the enhancement set by the Raman experiment. Because there is no acetone Raman signal detectable from the BP structure, Raman results alone will not allow a more exact comparison of theory with experiment.

Theoretical analysis ${ }^{17}$ suggests that Raman scattering can be modeled as resulting from the spontaneous emission of light at the Stokes frequency, where the molecules are driven by the pump field, with the frequency and intensity of the Raman response described by the Raman susceptibility tensor. Thus we can use another spontaneous process, PL, to estimate the actual Raman enhancement. We employed a specific fluorophore in water solution that can be excited at $780 \mathrm{~nm}$ and emits in the range of the investigated Stokes Raman signal. (We used the S0456 fluorophore from FEW CHEMICALS (3,3-Dimethyl-2[2-[2-chloro-3-[2-[1.3-dihydro-3,3-dimethyl-5-sulfo-1-(4-sulfobutyl)-" yl]-5-sulfo-1-(4-sulfobutyl)-3H-indolium hydroxide, inner salt, trisodium salt) that is optimized for absorbing at $\sim 780 \mathrm{~nm}: 2$ $\mu \mathrm{g}$ in $1 \mathrm{~mL}$ of distilled water.) The advantage of considering the corresponding PL experiment is that this process has a much larger cross section than Raman scattering; thus a detectable signal is expected for the bare prism. Although PL and Raman scattering are two different processes, the enhancement associated with the excitation of the BSW depends only on the optical properties of the samples at the pump and emitted light wavelengths. Thus, when working in the experimental configuration of Figure 4, the ratio between the PL signals measured from the multilayer and the bare prism should be a good estimate of the Raman enhancement.

The measured PL spectra for VIS and BP samples are shown in Figure 7. They are collected by means of the same apparatus utilized in the Raman measurements (that is, exciting the BSW with a TE-polarized pump beam and collecting the unpolarized light emitted in the cladding) but using a Mitutoyo $10 \times, \mathrm{NA}=$ 0.26 , microscope objective and working with a lower pump power, $\sim 100 \mu \mathrm{W}$, to avoid dye photobleaching. The PL of the VIS sample is $\sim 210$ larger than that of the BP sample. This value is on the same order of magnitude of what we expect from our theoretical analysis. The discrepancy can be ascribed to scattering and absorption losses, which are neglected in the calculations.

b. IR Sample. It is possible to obtain SERS working with pump wavelengths from visible to IR, using the same materials for the construction of the multilayer structures. For example,

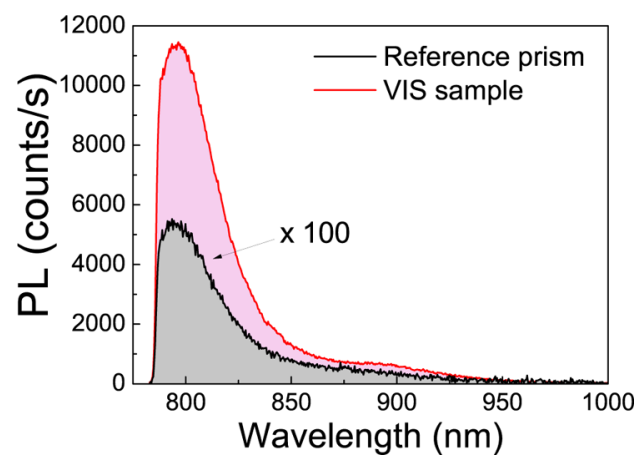

Figure 7. Photoluminescence intensity from BP and VIS sample for a solution of distilled water and $2 \mu \mathrm{g} / \mathrm{mL}$ of fluorophore.

this allows one to work at longer pump wavelengths to significantly reduce fluorescence background. A wide range of target materials can also be studied. To demonstrate this flexibility, we now turn to the IR sample, which has been designed to work with a pump at $1064 \mathrm{~nm}$, and we present the results of spontaneous Raman scattering from sulfur powder deposited on the multilayer surface.

We pump the IR sample with a Nd:YAG laser $(1064 \mathrm{~nm}$ Teem Photonics) at an external excitation angle $\theta_{\text {ext }} \approx 32^{\circ}$, which corresponds to the excitation of the BSW. The experimental configuration is conceptually the same as that in Figure 4. Here the pump laser is focused on the prism facet by a long focus lens $(f=100 \mathrm{~cm})$, with a spot on the multilayer surface with FWHM of 1 to $2 \mathrm{~mm}$. The incident power on the sample is $\sim 220 \mathrm{~mW}$. The scattered light is collected from the surface through a microscope objective (Olympus 10×, NA = 0.25 ), the Rayleigh component is filtered out by a long wave pass dichroic filter (Semrock LPD01-1064RU), and the signal is coupled into a multimode optical fiber and sent to a monochromator (SP-2500, Princeton Instruments) coupled to a silicon CCD (PIXIS-400, Princeton Instruments). Given the silicon CCD sensitivity, we use an integration time of 30 min to obtain the Raman spectra shown in Figure 8. Note that this configuration allows probing the sample response at different external angles while maintaining the same light collection conditions.

As before, we consider a bare N-BK7 prism as our reference to evaluate the Raman scattering enhancement. The Stokes Raman spectrum collected between 100 and $300 \mathrm{~cm}^{-1}$ is shown without background subtraction, in the main panel of Figure 8, where the two sulfur peaks at $\sim 150$ and $\sim 210 \mathrm{~cm}^{-1}$ are clearly visible. In the inset, we show the stronger Raman peak for the IR sample along with the Raman signal detected from the bare prism, where no peaks are visible. As in the previous case, we can estimate a lower bound for the enhancement only by dividing the intensity of the peak of the IR sample by the noise amplitude in the BP spectrum: this ratio is $\sim 50$.

\section{CONCLUSIONS}

In this work, we demonstrated SERS in fully dielectric structures supporting BSWs. This has been done by studying the Raman response of an aqueous solution as well as sulfur powder. We also showed that this approach allows us to work over a wide spectral region, the visible to IR, where the optical properties of the structure can be designed ad hoc without changing the constituent materials. 


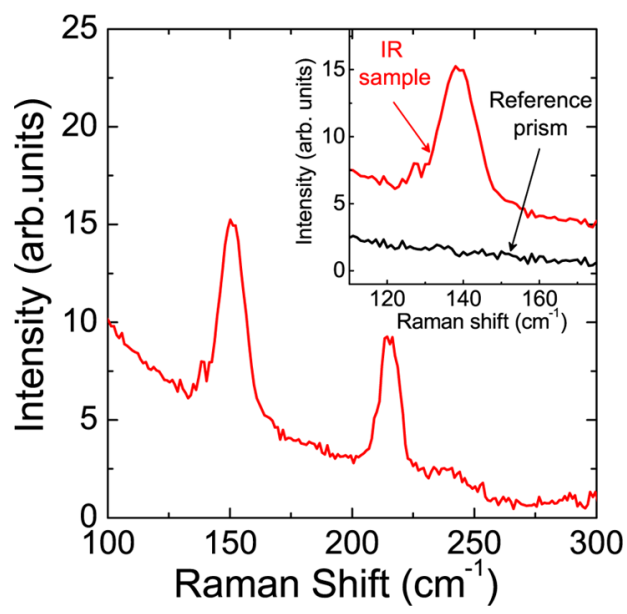

Figure 8. (Main panel) Raman spectrum for sulfur powder for the IR sample when the pump $\left(\lambda_{\text {pump }}=1064 \mathrm{~nm}\right)$ is resonantly coupled to the BSW. (Inset) Detail of the peak around $150 \mathrm{~cm}^{-1}$ (red line) and corresponding spectrum obtained in the same experimental condition for the bare N-BK7 prism (black line).

The theoretical model of Raman scattering suggests that the actual enhancement is indeed much larger: about 200-400 times. We showed that a similar enhancement is indeed observed in a PL experiment where a dye solution is excited by the same pump used in the Raman experiment and the light is collected in the same frequency range given by the Stokes shift in the Raman experiment.

These results demonstrate that dielectric multilayers supporting BSWs are a promising alternative to metallic structures for the realization of optical sensors based on Raman spectroscopy.

\section{AUTHOR INFORMATION}

\section{Corresponding Author}

*E-mail: stefano.pirotta01@ateneopv.it.

\section{Notes}

The authors declare no competing financial interest.

\section{ACKNOWLEDGMENTS}

This work was partially supported by the Natural Sciences and Engineering Research Council of Canada Strategic Network for Bioplasmonic Systems (BiopSys), and Regione Lombardia through "Fondo per la promozione di accordi istituzionali Progetti di cooperazione scientifica e tecnologica internazionale" (Project code SAL-11). Sudipta Maiti acknowledges support from DST, Govt. of India, grant no. ARCI/D/FAO/ Nano/K-16/Canada/11.

\section{REFERENCES}

(1) Moskovits, M. Surface-Enhanced Spectroscopy. Rev. Mod. Phys. 1985, 57, 783-826.

(2) Principles of Surface-Enhanced Raman Spectroscopy and Related Plasmonic Effects, 3rd ed.; Elsevier Science: Amsterdam, 2009.

(3) Campion, A.; Kambhampati, P. Surface-Enhanced Raman Scattering. Chem. Soc. Rev. 1998, 27, 241-250.

(4) Kambhampati, P.; Child, C. M.; Foster, M. C.; Campion, A. On the Chemical Mechanism of Surface Enhanced Raman Scattering: Experiment and Theory. J. Chem. Phys. 1998, 108, 5013-5026.

(5) Fleischmann, M.; Hendra, P.; McQuillan, A. Raman Spectra of Pyridine Adsorbed at a Silver Electrode. Chem. Phys. Lett. 1974, 26, $163-166$.
(6) Gersten, J. I. Rayleigh, Mie, and Raman Scattering by Molecules Adsorbed on Rough Surfaces. J. Chem. Phys. 1980, 72, 5780-5781.

(7) Brioude, A.; Lequevre, F.; Mugnier, J.; Dumas, J.; Guiraud, G.; Plenet, J. C. Raman Spectroscopy of Sol-gel Ultrathin Films Enhanced by Surface Plasmon Polaritons. J. Appl. Phys. 2000, 88, 6187-6191.

(8) Kahl, M.; Voges, E. Analysis of Plasmon Resonance and Surfaceenhanced Raman Scattering on Periodic Silver Structures. Phys. Rev. B 2000, 61, 14078-14088.

(9) Brolo, A.; Arctander, E.; Gordon, R.; Leathem, B.; Kavanagh, K. Nanohole-Enhanced Raman Scattering. Nano Lett. 2004, 4, 20152018.

(10) Nie, S.; Emory, S. R. Probing Single Molecules and Single Nanoparticles by Surface-Enhanced Raman Scattering. Science 1997, $275,1102-1106$

(11) Rabolt, J.; Santo, R.; Swalen, J. Raman Measurements on Thin Polymer Films and Organic Monolayers. Appl. Spectrosc. 1980, 34, 517-521.

(12) Rabolt, J.; Schlotter, N.; Swalen, J. Spectroscopic Studies of Thin Film Polymer Laminates Using Raman Spectroscopy and Integrated Optics. J. Phys. Chem. 1981, 85, 4141-4144.

(13) Kang, L.; Dessy, R. Slab Waveguide in Chemistry. Crit. Rev. Anal. Chem. 1990, 21, 377-388.

(14) Kanger, J.; Otto, C.; Greve, J. Stimulated Raman Gain Spectroscopy of Thin Layers Using Dielectric Waveguides. J. Phys. Chem. 1996, 100, 16293-16297.

(15) Pope, A.; Schulte, A.; Guo, Y.; Ono, L.; Cuenya, B.; Lopez, C.; Richardson, K.; Kitanovski, K.; Winningham, T. Chalcogenide Waveguide Structures as Substrates and Guiding Layers for Evanescent Wave Raman Spectroscopy of Bacteriorhodopsin. Vib. Spectrosc. 2006, 42, 249-253.

(16) Mak, J.; Farah, A.; Chen, F.; Helmy, A. Photonic Crystal Fiber for Efficient Raman Scattering of CdTe Quantum Dots in Aqueous Solution. ACS Nano 2011, 5, 3823-3830.

(17) Delfan, A.; Liscidini, M.; Sipe, J. Surface Enhanced Raman Scattering in the Presence of Multilayer Dielectric Structures. J. Opt. Soc. Am. B 2012, 29, 1863-1874.

(18) Yeh, P.; Yariv, A.; Cho, A. Optical Surface Waves in Periodic Layered Media. Appl. Phys. Lett. 1978, 32, 104-105.

(19) Liscidini, M.; Sipe, J. Analysis of Bloch-surface-wave Assisted Diffraction-based Biosensors. J. Opt. Soc. Am. B 2009, 26, 279-289.

(20) Liscidini, M.; Galii, M.; Shi, M.; Dacarro, G.; Patrini, M.; Bajoni, D.; Sipe, J. Strong Modification of Light Emission from a Dye Monolayer via Bloch Surface Waves. Opt. Lett. 2009, 34, 2318-2320.

(21) Liscidini, M.; Gerace, D.; Sanvitto, D.; Bajoni, D. Guided Bloch Surface Wave Polaritons. Appl. Phys. Lett. 2011, 98, 121118-121120.

(22) Descrovi, E.; Sfez, T.; Quaglio, M.; Brunazzo, D.; Dominici, L.; Michelotti, F.; Herzig, H.; Martin, O.; Giorgis, F. Guided Bloch Surface Waves on Ultrathin Polymeric Ridges. Nano Lett. 2010, 10, 2087-2091.

(23) Liscidini, M. Surface Guided Modes in Photonic Crystal Ridges: the Good, the Bad, and the Ugly. J. Opt. Soc. Am. B 2012, 29, 21032109.

(24) Galli, M.; Belotti, M.; Bajoni, D.; Patrini, M.; Guizzetti, G.; Gerace, D.; Agio, M.; Andreani, L.; Chen, Y. Excitation of Radiative and Evanescent Defect Modes in Linear Photonic Crystal Waveguides. Phys. Rev. B 2004, 70, 081307(1)-081307(4).

(25) Liscidini, M.; Galli, M.; Patrini, M.; Loo, R.; Goh, M.; Ricciardi, C.; Giorgis, F.; Sipe, J. Demonstration of Diffraction Enhancement via Bloch Surface Waves in a -SiN:H Multilayers. Appl. Phys. Lett. 2009, 94, 043117-043119.

(26) Spectrum Thin Films, Inc. http://www.spectrumthinfilms.com.

(27) Iridian, Spectral Technologies. http://www.iridian.ca. 